\title{
Equidad e inclusión en ambientes virtuales: un reto constante para la Institución Universitaria Politécnico Grancolombiano
}

\author{
Julián Andrés Arias-Vera ${ }^{1}$ \\ Institución Universitaria Politécnico Grancolombiano \\ jarias@poligran.edu.co \\ Sandra Milena Chicas-Sierra² \\ Institución Universitaria Politécnico Grancolombiano \\ smchicas@poligran.edu.co
}

DOI: https://doi.org/10.21158/2357514x.v9.n1.2021.3020

Cómo citar este artículo: Arias-Vera, J. A.; Chicas-Sierra, S. M. (2021). Equidad e inclusión en ambientes virtuales: un reto constante para la Institución Universitaria Politécnico Grancolombiano. Revista Virtu@lmente, 9(1), Páginas. DOI: https://doi.org/10.21158/2357514x.v9.n1.2021.3020

Fecha de recepción: 27 de mayo de 2021

Fecha de aprobación: 06 de agosto de 2021

\section{Resumen}

El presente artículo es un estudio exploratorio de la relación entre la educación superior virtual y la promoción de la equidad y la inclusión. Para esto se mide la percepción de los estudiantes del Politécnico Grancolombiano acerca de la promoción de equidad e inclusión educativa en sus programas académicos virtuales. La investigación se presenta mediante un enfoque cuantitativo haciendo uso de la encuesta como instrumento para la recolección de datos, correspondiente a un diseño no experimental y con alcance descriptivo. El Politécnico Grancolombiano cuenta con programas virtuales que han contribuido a la inclusión y educación de personas de regiones como Santander, Amazonas, Arauca, Casanare y Putumayo, entre otras, lo cual evidencia el gran impacto de la institución en la población vulnerable y excluida, además, los resultados muestran que la institución realiza actividades de formación para profesionales con diversas identidades culturales, orientada a la excelencia, el respeto por la diferencia, las oportunidades, la libre interacción y comunicación, así como la posibilidad de acceder a mejores oportunidades laborales con niveles superiores al $90 \%$, por lo que cumple con lo establecido en su proyecto educativo institucional. Finalmente, se concluye la importancia de continuar generando actividades que promuevan la equidad e inclusión y lleven la generación de accesibilidad y oportunidades para todos, específicamente en lo que tiene que ver con los retos hacia el mercado laboral.

Palabras clave: educación superior virtual; programas de formación virtual; educación accesible; ambientes virtuales de aprendizaje; inclusión social; educación para población vulnerable.

\footnotetext{
${ }^{1}$ Administrador de empresas - Universidad Nacional sede Manizales. Especialista en Finanzas Corporativas - Universidad Nacional sede Manizales. Especialista en Alta Gerencia - Corporación Universitaria Remington. Especialista en Herramientas virtuales para la educación - Politécnico Grancolombiano. ORCID: https://orcid.org/0000-0003-2962-1516

Magister en Administración con especialidad en Finanzas Corporativas - Universidad Viña del Mar

${ }^{2}$ Administradora de empresas- Universidad del Norte. Especialista en gerencia de comercio internacional- Universidad Militar Nueva Granada. Magíster en Comercio Internacional- Universidad Sergio Arboleda. Estudiante de Doctorado en Sociedad del conocimiento- Universidad Internacional de la Rioja. ORCID: https://orcid.org/0000-0002-4572-9132
} 
Equity and inclusion in virtual environments:

a constant challenge for Institución Universitaria Politécnico Grancolombiano

\begin{abstract}
This article is an exploratory study of the relationship between virtual higher education and the promotion of equity and inclusion. For this purpose, it measures the perception of the students of the Politécnico Grancolombiano about the promotion of equity and educational inclusion in its virtual academic programs. The research adopts a quantitative approach using the survey as an instrument for data collection, corresponding to a non-experimental design and with a descriptive scope. The Politécnico Grancolombiano has virtual programs that have contributed to the inclusion and education of people from regions such as Santander, Amazonas, Arauca, Casanare, and Putumayo, among others, evidencing the great impact of the institution on the vulnerable and excluded population; in addition, the results show that the institution carries out training activities for professionals with diverse cultural identities, oriented to excellence, respect for difference, opportunities, free interaction and communication, as well as the possibility of accessing better job opportunities with levels above $90 \%$, thus complying with its institutional educational project. Finally, we emphasize the importance of continuing to generate activities that promote equity and inclusion, and lead to the development of accessibility and opportunities for all, specifically in what has to do with the challenges towards the labor market.
\end{abstract}

Keywords: virtual higher education; virtual training programs; accessible education; virtual learning environments; social inclusion; education for vulnerable population.

Equidade e inclusão em ambientes virtuais:

um desafio constante para a Instituição Universitária Politécnico Grancolombiano

\title{
Resumo
}

Este artigo é um estudo exploratório da relação entre o Ensino Superior virtual e a promoção da equidade e inclusão. Para isso, mede-se a percepção dos alunos do Politécnico Grancolombiano sobre a promoção da equidade e da inclusão educacional em seus programas acadêmicos virtuais. A pesquisa é apresentada por meio de uma abordagem quantitativa utilizando questionários como instrumento de coleta de dados, correspondendo a um desenho não experimental e de abrangência descritiva. O Politécnico Grancolombiano possui programas virtuais que têm contribuído para a inclusão e educação de pessoas de regiões como Santander, Amazonas, Arauca, Casanare e Putumayo, entre outras, o que mostra o grande impacto da instituição na população vulnerável e excluída, além disso, os resultados mostram que a instituição realiza atividades de formação para profissionais com identidades culturais diversas, orientadas para a excelência, respeito à diferença, oportunidades, livre interação $e$ comunicação, bem como a possibilidade de acesso a melhores oportunidades de trabalho com níveis superiores a 90\%, portanto, está de acordo com o que está estabelecido em seu projeto educacional institucional. Finalmente, conclui-se a importância de continuar a gerar atividades que promovam a equidade e inclusão e levem à geração de acessibilidades e oportunidades para todos, especificamente no que se refere aos desafios do mercado de trabalho.

Palavras-chave: Ensino Superior virtual; programas de formação virtual; educação acessível; ambientes virtuais de aprendizagem; inclusão social; educação para a população vulnerável. 
Équité et inclusion des environnements virtuels d'apprentissage: un défi constant pour l'Institution universitaire polytechnique Grancolombiano

\begin{abstract}
Résumé
Cette étude explore les relations entre l'enseignement supérieur virtuel et la promotion de l'équité et de l'inclusion. Nous mesurerons la perception des étudiants de l'institut polytechnique Grancolombiano sur la promotion de l'équité et de l'inclusion éducative dans leurs programmes académiques virtuels. L'étude est réalisée sous une approche quantitative de collecte de données correspondant à une conception descriptive et non expérimentale. L'institut polytechnique Grancolombiano dispose de programmes virtuels contribuant à l'insertion professionnelle et à l'éducation universitaire des étudiants des régions de Santander, Amazonas, Arauca, Casanare et Putumayo, révélant l'impact de l'institution sur les populations vulnérables. Les résultats de l'étude montrent également que l'institution mène des activités de formation pour des apprenants d'identités culturelles diverses, que ces formations sont tournées vers l'excellence, le respect des différences, la libre interaction et la communication, et la possibilité d'accéder à de meilleures opportunités d'emploi. C'est en cela que l'institut Grancolombiano respecte le cadre de son projet éducatif institutionnel, en se focalisant sur l'importance de continuer à générer des activités favorisant l'équité et l'inclusion, conduisant à la création d'opportunités pour tous, en particulier pour les nouveaux défis auxquels le marché du travail se trouve confronté.
\end{abstract}

Mots-clés: enseignement supérieur virtuel; programmes de formation virtuels; éducation accessible; environnements d'apprentissage virtuels; inclusion sociale; éducation des populations vulnérables.

\title{
1. Introducción
}

La educación es un derecho fundamental del ser humano, un requisito esencial para reducir la pobreza (Navarro y Martínez de Anguita, 2008). De acuerdo con la Unesco, un país se considera libre de analfabetismo cuando el $4 \%$ o menos de su población es analfabeta. Sin embargo, según la Misión de Ciencia, Educación y Desarrollo, como se cita en Chona-Duarte (1998), la tasa de analfabetismo en Colombia es del 5,8\%.

Esto significa que, de los 46 millones de habitantes, según el último censo nacional del Departamento Administrativo Nacional de Estadística (DANE, 2019), 27000000 habitantes se encuentran en situación de analfabetismo, de los cuales el $92 \%$ está concentrado en departamentos como La Guajira, Cesar, Magdalena, Sucre, Córdoba, Chocó, Vichada, Guainía, Amazonas, Putumayo y Vaupés. En este sentido, la educación virtual se propone como estrategia para mejorar los índices de alfabetización, inclusión y equidad. 
Este escrito tiene como propósito medir la percepción de los estudiantes en materia de equidad e inclusión desde los programas virtuales de la Institución Universitaria Politécnico Grancolombiano. Para cumplir con este fin, se diseñó y aplicó una encuesta en escala de Likert, sometida a validez por un equipo experto, con el objetivo de conocer la percepción de los estudiantes frente al tema propuesto y adoptar un diseño no experimental.

El alcance estaría dado por el análisis de los datos tomados de las encuestas contestadas; la limitación es que la encuesta se aplicó a los estudiantes que actualmente estaban inscritos en los pregrados virtuales que pretendían estudiar. La población objeto de estudio es de 200 estudiantes de los programas de Negocios Internacionales y Administración de Empresas de la modalidad virtual del Politécnico Grancolombiano, correspondientes a las asignaturas Introducción al Comercio Internacional y Procesos Administrativos.

\section{Antecedentes}

\subsection{Consideraciones sobre la población vulnerable en Colombia}

Históricamente, en Colombia el mayor problema del sector educativo ha sido la deserción, entendida como «la interrupción o desvinculación de los estudiantes de sus estudios» (Ministerio de Educación Nacional, s.f.), especialmente en personas que viven en áreas rurales y apartadas, una población conformada, principalmente, por indígenas, campesinos y afrodescendientes. Las zonas rurales del país presentan graves problemas asociados al desempleo, la extrema pobreza y el analfabetismo, entre otros, lo que dificulta la permanencia de los niños en las escuelas, tal y como se enuncia a continuación. 
De 100 estudiantes que se matriculan en primero de primaria en las zonas rurales, solo 35 terminan este ciclo y un poco menos de la mitad -16 estudiantes - pasan a secundaria; de estos, 8 completan el noveno grado y solo 7 culminan el ciclo completo de educación básica. (Perfetti, 2004, p. 183)

Lo anterior, sin contar con la falta de infraestructura, la falta de materiales didácticos, laboratorios, el acceso inexistente a internet y mucho menos a computadores, sumado a las precarias vías que deben recorrer los niños que desean llegar a la escuela más cercana. Estas circunstancias hacen que la situación de la población rural e indígena se encuentre en condiciones de exclusión y vulnerabilidad. La brecha entre la ciudad y el campo es cada vez mayor, lo que genera altos índices de desigualdad y exclusión social, como, por ejemplo, en la región del Chocó.

El ingreso por habitante chocoano medio es la sexta parte del de un bogotano. Una de las verdades que no encara la política pública en Colombia es el desequilibrio entre las diez principales ciudades, que crecen y son polos de desarrollo y 800 municipios que tienen niveles de pobreza superiores al 66 por ciento. (Desigualdad Extrema, 2011, pár. 8)

Desde la educación superior, la deserción es un problema que afecta a todas las instituciones superiores a nivel global, en especial a la población en condición de vulnerabilidad, con un serio déficit de oferta educativa. Esto se debe a que las universidades y demás instituciones educativas se encuentran en grandes ciudades de Colombia, lo que dificulta el acceso a la educación para las personas que viven en áreas rurales (Gutiérrez-Ortiz, García, Barrientos-Monsalve y Jejen, 2018).

Sumado a esto, la deserción educativa también puede darse por las características de la población vulnerable, que incluye personas con limitaciones para ver, caminar u oír. Colombia cuenta con un $6,4 \%$ de población en estas condiciones especiales, concentradas en los departamentos de Cauca $-9,5 \%$-y Tolima $-7,8 \%$ - (Rojas-Rojas, Arboleda-Toro y Pinzón-Jaime, 2018). 
Así mismo, no se puede dejar de lado la población en condición de vulnerabilidad como consecuencia del conflicto armado que vivió el país, lo cual provocó que mucha población rural se desplazara hacia las grandes ciudades, lo que impactó de manera negativa su acceso a la educación; por esta razón, la inclusión social mediante las nuevas tecnologías de información presenta una oportunidad para cerrar la brecha de exclusión dada por las circunstancias del posconflicto (Loaiza-Álvarez, 2017).

Hoy en día, el Gobierno colombiano ha impulsado propuestas para mejorar el sistema educativo y, en especial, mejorar la cobertura y el acceso a la educación; sin embargo, a pesar de estos esfuerzos, «los logros en cuanto a cobertura y calidad educativa en el país siguen estando rezagados» (García, Maldonado y Rodríguez, 2014, p. 1). De acuerdo con el último informe presentado por el Ministerio de Educación Nacional, para el 2017 hubo un total de 463457 graduados de educación superior en todo el país, el valor por regiones se presenta en la figura 1.

Figura 1. Caracterización de graduados de educación superior por departamento año 2017

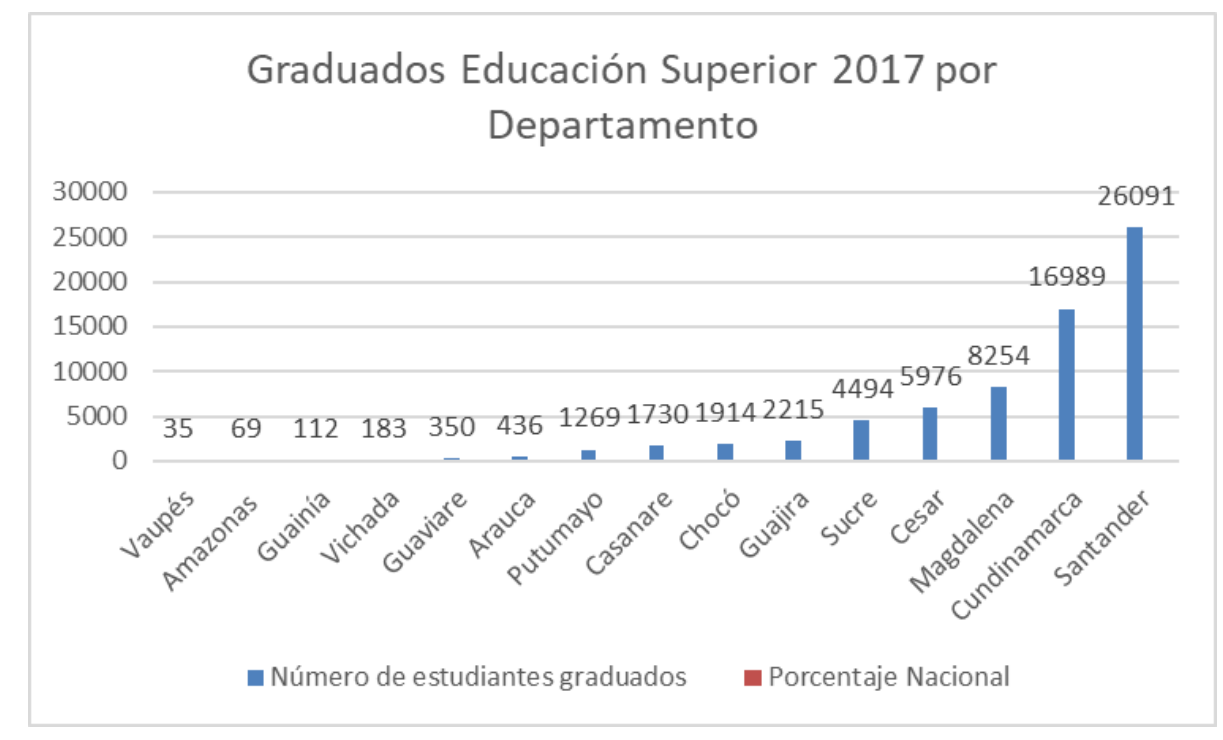

Fuente. Elaboración propia con base en información del Ministerio de Educación Nacional. 
Como se puede observar, la mayor concentración de la población de graduados en educación superior proviene de los departamentos de Antioquia, Valle del Cauca, Santander y Cundinamarca, con un número menos importante en los departamentos de Magdalena, Cesar y Sucre. Las cifras son sumamente críticas en las regiones del Vaupés, Amazonas, Guainía, Vichada, Guaviare, Arauca, Putumayo, Casanare, Chocó y La Guajira.

Ahora bien, el Ministerio de Educación Nacional (MEN) identifica como población vulnerable a «aquellas personas que, por su naturaleza o por determinadas circunstancias se encuentren en mayor medida expuestos a la exclusión, la pobreza y los efectos de la inequidad y la violencia de todo orden» ( Ministerio de educación Nacional, 2005, p. 20); esto deja en evidencia la situación de las regiones expuestas y el bajo número de graduados, dada su connotación.

De acuerdo con el Ministerio de Salud de Colombia, la población vulnerable está dividida en dos grandes grupos. El primero, en función del curso de vida incluye niños, niñas, adolescentes, jóvenes, adultos y adultos mayores; el segundo, correspondiente a poblaciones con condiciones o situaciones particulares como son los grupos étnicos, la población en situación de discapacidad y las víctimas del conflicto armado (Ministerio de Salud, s. f.).

\subsection{La inclusión social por medio de la educación virtual}

Para entender el concepto de inclusión social primero se debe entender el concepto de exclusión social. Hablar de exclusión social no solo implica pobreza económica, también se puede hablar de exclusión social cuando hay falta de oportunidades laborales, de acceso a educación o a salud y vivienda (Arroyo-González, 2013). Bajo este contexto, se

puede afirmar que la inclusión está relacionada con el acceso, la participación y los logros 
de todos los alumnos, con especial énfasis en aquellos que están en riesgo de ser excluidos o marginados.

Aclarado el concepto de inclusión social, se puede hablar de educación inclusiva. Este concepto implica hacer que el estudiante se sienta aceptado, reconocido y valorado en su entorno educativo, sin importar su género, raza, religión o estrato social. Este concepto aplica no solo para mujeres, hombres y niños de nivel socioeconómico pobre, sino para personas con alguna discapacidad que viven en áreas rurales o simplemente personas que son inmigrantes.

En ese sentido, Echeita-Sarrionandia (2008) afirma que «la educación inclusiva debe entenderse con igual fuerza como la preocupación por un aprendizaje y un rendimiento escolar de calidad y exigente con las capacidades de cada estudiante» (p. 11). Esto implica que las instituciones educativas deben plantear una metodología pedagógica innovadora que no solo facilite la adquisición de conocimiento, sino que, además, motive a los estudiantes a participar en las clases, a trabajar en equipo, crear autodisciplina, enfocados, finalmente, en conseguir un alto nivel de rendimiento educativo. Así, pues, el diseño de los ambientes de aprendizaje, según Guerrero-Dávila y Páramo-Herrera (2015), debe tener en cuenta aspectos tales como la diversidad humana, la equidad, la igualdad y la inclusión para cumplir con el propósito de disminuir los índices de exclusión social y económica.

Ahora bien, el concepto de educación inclusiva está reglamentado por la Unesco como un derecho fundamental y como el camino hacia el futuro, especialmente en países en desarrollo. Al tomar como base que la educación es un derecho fundamental, la educación inclusiva debería considerarse una política de Estado en todos los gobiernos y ser prioridad en países en desarrollo: «estamos hablando, por tanto, de una investigación inclusiva, democrática y socialmente justa» (Murillo y Duk, 2018, p. 3). 
Este proceso debe estar encaminado a entender la diversidad cultural de los estudiantes, para, de esa manera, plantear estrategias educativas en concordancia con las competencias y necesidades de la comunidad, tal lo explican Hurtado-Lozano y AgudeloMartínez (2014): «la educación inclusiva se entiende como una búsqueda incesante de mejores formas de responder a la diversidad» (p. 48).

En este punto cabe resaltar que la educación inclusiva debe basarse en un modelo de educación de calidad. De acuerdo con la Unesco, en su informe de seguimiento de educación para todos en el mundo, la educación de calidad se define bajo tres parámetros: el respeto de los derechos de las personas, la equidad en el acceso, los procesos y resultados y la pertinencia de la educación (Unesco, 2019). No obstante, las instituciones de educación superior deben garantizar la pertinencia de los programas académicos, ya que, así, el estudiante se sentirá identificado y motivado, de manera que se reflejen sus resultados de aprendizaje en la práctica. Es por esto que el proceso de inclusión a través de la educación debe darse por medio de estrategias pedagógicas que atiendan las necesidades y el contexto sociocultural de cada individuo (Beltrán-Villamizar, MartínezFuentes y Vargas-Beltrán, 2015).

Para el Ministerio de Educación Nacional de Colombia, la educación inclusiva es la posibilidad de acoger en la institución educativa a todos los estudiantes, independientemente de sus características personales, culturales, cognitivas y sociales. En este sentido, el Ministerio de Educación Nacional de Colombia plantea seis características de la educación inclusiva: la participación, la diversidad, la pertinencia, la calidad, la equidad y la interculturalidad (Ministerio de educación Nacional, 2009). Estas características deben reflejarse en los proyectos educativos institucionales de cada universidad o centro educativo, con el fin de evaluar si desarrollan actividades que promuevan la equidad y la inclusión desde las aulas. 
Así, pues, educar en la universidad de hoy busca enseñar a adaptarse a los cambios que se generan $y$, sin duda, con miras a la inclusión «la adopción de la tecnología en los procesos de enseñanza ha permitido dar paso de la presencialidad a la virtualidad creando un sinnúmero de recursos, entornos, herramientas y ambientes, para el aprendizaje en red que facilitan la tarea» (Arias-Vera et al., 2018).

En ese mismo sentido, en los últimos años se ha hablado de la educación virtual como estrategia para la inclusión social, ya que por medio de la educación virtual inclusiva se atiende no solo la diversidad de género, sino a personas de diferentes razas, minorías étnicas, desplazados y demás población en condición de vulnerabilidad (MondragónHernández, 2018).

Durán-Gisbert y Giné-Giné (2017) expresan la preocupación porque la escuela inclusiva se ha convertido en uno de los mayores retos que actualmente deben afrontar los sistemas educativos, los centros, el profesorado y la sociedad. Por tanto, se debe garantizar que la educación virtual sea de calidad y así cerrar la brecha en materia de desigualdad, ya que el perfil de los estudiantes de programas virtuales puede incluir desde el alto ejecutivo que no tiene mucho tiempo para asistir a clases presenciales, hasta la madre cabeza de hogar que trabaja de día y estudia en sus ratos libres mientras sus hijos duermen.

De igual manera, Cabrero-Almenara (2016) expone que, a través de la educación virtual inclusiva, las instituciones educativas contribuyen al empoderamiento intelectual al aumentar la equidad y la justicia social, en el marco del derecho fundamental a la educación. Por medio de la educación virtual los estudiantes desarrollan nuevas formas de convivencia y trabajo en equipo, al tiempo que descubren sus propias habilidades.

La inclusión educativa, vista como un derecho fundamental, debe garantizar la calidad y la equidad en los servicios, así como la atención ofrecidos por las instituciones educativas, 
además de privilegiar la diversidad sobre la homogeneidad, teniendo en cuenta que no todos los estudiantes aprenden de la misma forma (Materón-Palacios, 2016). De ahí que la educación virtual se considere importante en el desarrollo socioeconómico, laboral y educativo de los países, porque exige romper paradigmas relacionados con metodologías de educación, generando una transformación en los modelos pedagógicos de enseñanzaaprendizaje y en los modelos de interacción bajo ambientes virtuales (Vilanova, 2016).

Lo anterior es fundamental en países como Colombia, donde existe un alto grado de analfabetismo rural debido, en gran parte, a temas como la gratuidad de la educación, la igualdad de acceso, la atención a la diversidad y el acceso sin distinción de raza o género (Cabrero-Almenara, 2016). La educación virtual inclusiva permite que los estudiantes puedan potenciar sus habilidades para impactar positivamente en el desarrollo y la productividad del país.

Así las cosas, el tema de la educación virtual es relevante hoy en día, ya que el avance en la tecnología y la globalización permite ahora que muchas más personas en el mundo puedan acceder a la educación sin trasladarse, interactuando y manejando de manera flexible su tiempo de estudio; solo basta con tener una buena conexión a internet, una tableta, un computador o un smartphone y disciplina (Hernández, Villa y Vázquez, 2017).

La educación virtual se presenta hoy en día como una tendencia tecnológica y cultural que sobrepasa el ámbito de la educación escolar. En consecuencia, la educación basada en tecnologías de la información y la comunicación (TIC) se concibe como una herramienta que brinda soluciones al problema de la exclusión, porque permite que las personas se entrenen en habilidades tecnológicas y digitales para incorporarse correctamente a la sociedad del conocimiento; en fin, la educación virtual es un aliado en la inclusión socioeconómica (Rueda-Ortíz y Franco-Avellaneda, 2018). 
En este punto cabe resaltar que la educación basada en el uso de las tecnologías de la información y las comunicaciones tiene un impacto en el estudiante porque lo incentiva a estar en constante actualización y crecimiento profesional, fomenta el aprendizaje interactivo, además es flexible y reduce la brecha geográfica, especialmente en países como Colombia, considerado una economía emergente. De esta manera, es responsabilidad de las instituciones educativas crear entornos virtuales de aprendizaje con el ánimo de fomentar la cooperación y las prácticas pedagógicas inclusivas (Calvo, 2018). Por esta razón, la educación virtual debe ir en concordancia con las exigencias del entorno económico, social y político, y se considera una nueva perspectiva pedagógica.

Hoy en día, en Colombia es posible encontrar no solo programas de pregrado y posgrado virtuales, sino de educación continuada, como, por ejemplo, los diplomados o cursos cortos, que mejoran las oportunidades de los profesionales; esto generó un aumento en el número de instituciones educativas que están desarrollando programas de educación virtual (Ministerio de Educación Nacional, 9 de julio 2009).

El Politécnico Grancolombiano, por ejemplo, ofrece programas de pregrado y posgrado en modalidad virtual, impulsados por el Decreto 1421 de 2017: Por el cual se reglamenta en el marco de la educación inclusiva la atención educativa a la población con discapacidad, indudablemente en coherencia con lo que propone el sector de la educación en el marco de educación inclusiva y de calidad.

El compromiso de la institución frente a la promoción de la equidad y la inclusión se puede evidenciar en el Proyecto Educativo Institucional (PEI), en el cual se resalta que el mayor impacto de los programas virtuales se ha dado en el aumento del número de estudiantes de diversas poblaciones y características tales como la edad, las condiciones socioeconómicas y la ubicación geográfica. 
El Politécnico Grancolombiano respeta las diferencias individuales en relación con el género, la filiación sexual, religiosa o política, por lo que brinda diversas alternativas para que los estudiantes puedan terminar sus estudios; algunas de estas alternativas incluyen becas, combinar cursos presenciales y virtuales, cursar los programa en diferentes etapas y moverse dentro de los niveles superiores de formación, de forma horizontal o vertical, sin perder lo que se ha cursado (Politécnico Grancolombiano, 2020).

\section{Problema de investigación}

Dado el contexto anterior, es necesario medir la percepción de los estudiantes frente a la promoción de la educación equitativa e inclusiva y la contribución de la virtualidad en la mejora del acceso a la educación en zonas con población vulnerable. Entendiendo que el modelo de educación virtual es una herramienta importante para la inclusión social, ya que permite el acceso al aprendizaje y a la información, al crear escenarios de aprendizaje acordes con las necesidades de la comunidad y adaptándose a los diferentes estilos de aprendizaje, se plantea la siguiente pregunta de investigación: ¿la Institución Universitaria Politécnico Grancolombiano promueve la equidad e inclusión desde sus programas virtuales de educación superior?

\section{Metodología}

La presente investigación se enmarca en el proyecto de tesis doctoral que está actualmente en ejecución por uno de los autores, en torno al tema central aquí propuesto. Su diseño es de orden no experimental con alcance descriptivo, cuyo instrumento fue una encuesta aplicada a estudiantes de pregrado virtual de dos programas académicos de la institución educativa. 


\subsection{Descripción del contexto y la muestra}

Los autores del estudio son profesores de la facultad de Negocios, Gestión y Sostenibilidad de la Institución Universitaria Politécnico Grancolombiano que, durante el 2018, orientaron un total de seis grupos virtuales en asignaturas de los programas de Administración de Empresas y Negocios Internacionales. Al tomar en cuenta que la institución cuenta con 50000 estudiantes activos, distribuidos en diferentes regiones, tal como lo muestra la figura 1, se seleccionó un total de 200 estudiantes de regiones sobre las que se pudiera cumplir con el propósito de la investigación y que, además, cursaron las asignaturas de Negociación Internacional y Procesos Administrativos bajo la modalidad virtual.

Figura 2. Estudiantes modalidad virtual por región Politécnico Grancolombiano 2018-01

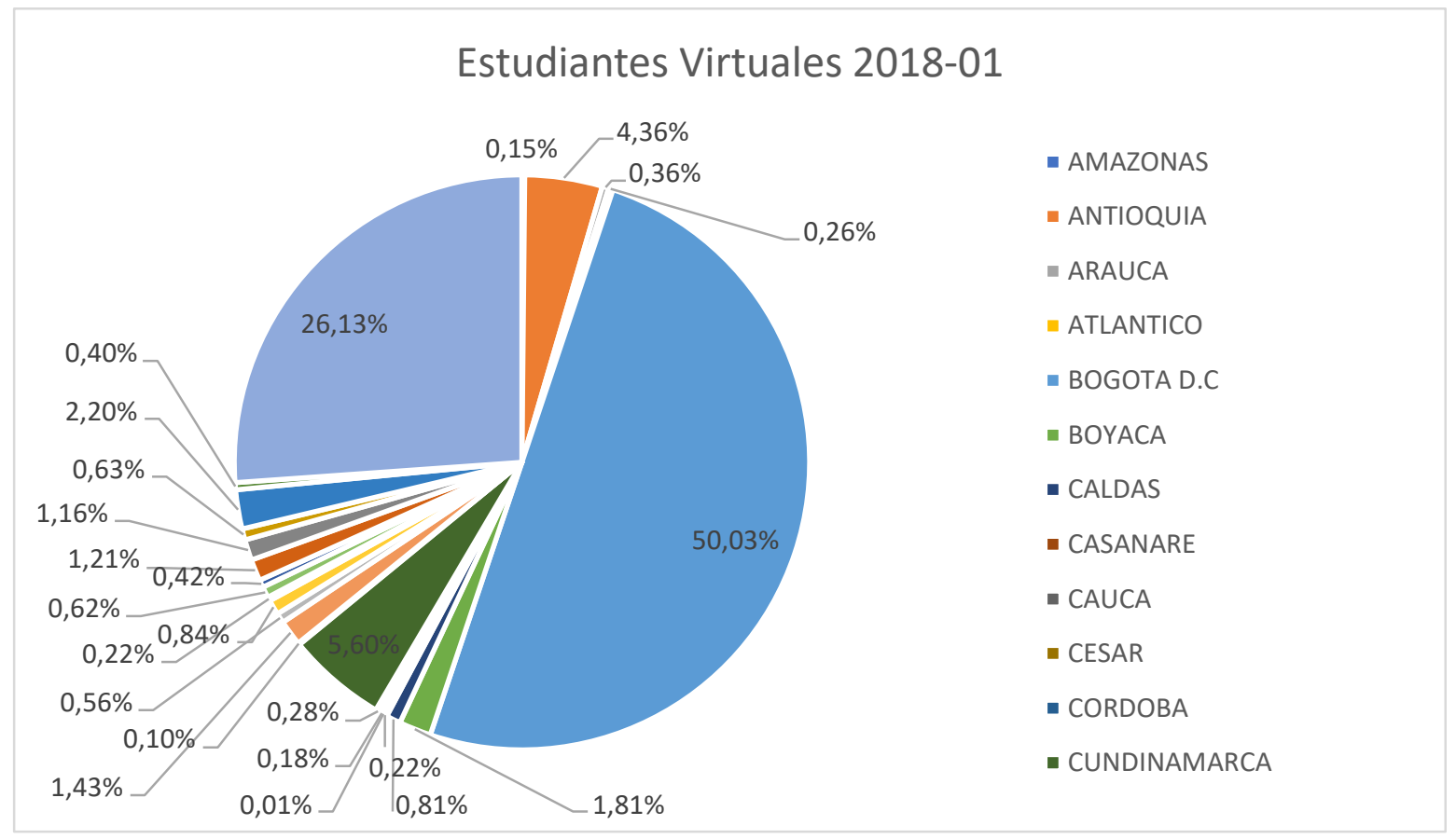

Fuente. Elaboración propia sobre información de matrículas del Politécnico Grancolombiano. 


\subsection{Instrumento}

La recolección de datos inicialmente se concentró en recopilar información de edad y sexo de los estudiantes, para luego aplicar un instrumento único, una encuesta de percepción del alumnado con siete variables, seis de ellas son las características de la educación inclusiva propuestas por el Ministerio de Educación Nacional —participación, diversidad, pertinencia, calidad, equidad e interculturalidad-y la variable restante indaga sobre la empleabilidad, todas ellas en escala Likert —calificación de 1 a 5-.

\subsection{Procedimiento}

La investigación sucedió en varias fases: en un primer momento se realizó una revisión documental en materia de inclusión, ambientes virtuales y educación superior, para luego en un segundo y tercer momento realizar el diseño y la elaboración del instrumento, así como la selección de la muestra, respectivamente; en cuarto lugar, se procedió a la recolección de los datos para en un quinto momento procesar la información recopilada y proceder con la elaboración de las conclusiones y, finalmente, exponer y divulgar los resultados del estudio realizado.

El procedimiento para la obtención de los datos se generó al contactar a los estudiantes de las asignaturas de negociación internacional y los procesos administrativos, se les informó de la realización del estudio, señalando el objetivo de la investigación, para luego consultar acerca de su intención de participación de forma voluntaria, aclarando que los datos serían tratados de forma anónima y que la información recolectada sería analizada no de forma individual, sino agregada. Después de informados los estudiantes, se realizó la aplicación del instrumento de forma online durante el periodo comprendido entre junio y agosto del 2018. 


\section{Resultados}

La realización de la indagación de datos descriptivos permite inferir que en el estudio participaron 130 mujeres - $65 \%$ - y 70 hombres - $35 \%$ - con una edad promedio en mujeres de 30 años y en hombres de 27; en general, el promedio de edad de los encuestados es de 29 años, pertenecientes a las regiones que se muestran en la tabla 1.

Tabla 1. Estudiantes por departamento/estado que dieron respuesta al instrumento

\begin{tabular}{|c|c|}
\hline Departamento/Estado & N. ${ }^{\circ}$ de estudiantes \\
\hline Amazonas & 9 \\
\hline Meta & 11 \\
\hline Antioquia & 24 \\
\hline San Andrés Islas & 10 \\
\hline Huila & 15 \\
\hline Casanare & 17 \\
\hline Vichada & 25 \\
\hline Vaupés & 2 \\
\hline Cauca & 22 \\
\hline Putumayo & 34 \\
\hline Norte de Santander & 11 \\
\hline Villavicencio & 16 \\
\hline Emiratos Árabes Unidos & 1 \\
\hline Houston Texas & 1 \\
\hline Madrid - España & 1 \\
\hline Risaralda & 1 \\
\hline Total & $\mathbf{2 0 0}$ \\
\hline
\end{tabular}

Fuente. Elaboración propia.

Como se puede evidenciar en la tabla 1, los estudiantes encuestados pertenecen a diversas regiones de nuestro país, algunas de ellas mencionadas como zonas geográficas donde los niveles de alfabetización y las oportunidades de cursar programas en educación superior son mínimas, lo que demuestra el esfuerzo que la institución ha venido realizando 
por hacer presencia en las regiones. En algunos casos, evidentemente de menor proporción, el instrumento fue diligenciado por estudiantes que residen en el exterior, en países como España, Emiratos Árabes y Estados Unidos, lo que permite determinar que el alcance de la educación virtual no distingue fronteras específicas.

En cuanto al instrumento, las siete preguntas planteadas y formuladas bajo escala Likert - del 1 al 5- se plantearon de forma cerrada y categorizadas con las afirmaciones siempre -calificación 5-, casi siempre —calificación 4-, ocasionalmente -calificación 3-, casi nunca -calificación 2- y nunca -calificación 1-. Las dimensiones y afirmaciones del instrumento se enlistan a continuación.

A. Participación: la institución promueve la intervención y expresión de ideas, así como en la invitación de grupos, redes y eventos, en general a actividades.

B. Diversidad: la institución acepta y promueve la diversidad y la diferencia en un sentido humano y social.

C. Pertinencia: la institución, en general, promueve oportunidades adecuadas y convenientes para todas las personas, sin distinción alguna.

D. Calidad: la institución promueve una inclusión orientada a la excelencia, satisfaciendo las necesidades de todos sus estudiantes.

E. Equidad: la institución otorga e incluye a las personas de acuerdo con su mérito, sin favorecer a una persona y perjudicar a otra.

F. Interculturalidad: se promueve la interacción y la comunicación entre personas de diversas culturas. 


\section{G. Empleabilidad: cursar programas virtuales en la institución ha permitido generar nuevas oportunidades laborales.}

La proporción de respuestas a las dimensiones abordadas se presentan en la tabla 2.

Tabla 2. Proporción de respuestas al instrumento aplicado

\begin{tabular}{|c|c|c|c|c|c|c|}
\hline Dimensiones & Criterio & 5 & 4 & 3 & 2 & 1 \\
\hline Participación & $\begin{array}{l}\text { La institución promueve la } \\
\text { intervención y expresión de ideas, así } \\
\text { como en la invitación de grupos, } \\
\text { redes y eventos, en general a } \\
\text { actividades. }\end{array}$ & $43,9 \%$ & $38,5 \%$ & $15,0 \%$ & $1,1 \%$ & $1,5 \%$ \\
\hline Diversidad & $\begin{array}{l}\text { La institución acepta y promueve la } \\
\text { diversidad y la diferencia en un } \\
\text { sentido humano y social. }\end{array}$ & $59,4 \%$ & $33,7 \%$ & $6,4 \%$ & $0,0 \%$ & $0,5 \%$ \\
\hline Pertinencia & $\begin{array}{l}\text { La institución en general promueve } \\
\text { oportunidades adecuadas y } \\
\text { convenientes para todas las } \\
\text { personas, sin distinción alguna. }\end{array}$ & $62,6 \%$ & $30,5 \%$ & $6,9 \%$ & $0,0 \%$ & $0,0 \%$ \\
\hline Calidad & $\begin{array}{l}\text { La institución promueve una } \\
\text { inclusión orientada a la excelencia, } \\
\text { satisfaciendo las necesidades de } \\
\text { todos sus estudiantes. }\end{array}$ & $57,8 \%$ & $34,8 \%$ & $6,9 \%$ & $0,0 \%$ & $0,5 \%$ \\
\hline Equidad & $\begin{array}{l}\text { La institución otorga e incluye a las } \\
\text { personas de acuerdo con su mérito, } \\
\text { sin favorecer a una persona y } \\
\text { perjudicar a otra. }\end{array}$ & $55,6 \%$ & $34,8 \%$ & $4,3 \%$ & $3,2 \%$ & $2,1 \%$ \\
\hline Interculturalidad & $\begin{array}{l}\text { Se promueve la interacción y la } \\
\text { comunicación entre personas de } \\
\text { diversas culturas. }\end{array}$ & $64,7 \%$ & $23,0 \%$ & $10,7 \%$ & $1,1 \%$ & $0,5 \%$ \\
\hline Empleabilidad & $\begin{array}{l}\text { Cursar programas virtuales en la } \\
\text { institución ha permitido generar } \\
\text { nuevas oportunidades laborales. }\end{array}$ & $58,5 \%$ & $36,0 \%$ & $2,8 \%$ & $1,1 \%$ & $1,6 \%$ \\
\hline
\end{tabular}

Fuente. Elaboración de los autores con base en la población objeto de estudio. 
Sobre la dimensión A en materia de participación se establece que la institución, en un 43,9\%, siempre promueve la interacción y expresión de ideas. En relación con la dimensión B que implica la diversidad, se establece que la institución acepta y promueve la diversidad y la diferencia en un 59,4 \%. La dimensión C, por su parte, obtiene un 62,6 \% de respuesta favorable, lo cual evidencia que se promueven oportunidades adecuadas y convenientes a todas las personas, sin distinción alguna. En cuanto a la percepción de la calidad - dimensión D-, el 57,8 \% de los alumnos afirma que siempre se promueve la calidad satisfaciendo necesidades de todos los estudiantes, mientras que para la dimensión de equidad —criterio $\mathrm{E}$ - el 55,6 \% de la muestra respondió que la institución otorga e incluye a las personas de acuerdo con su mérito, sin favorecer a una persona y perjudicar a otra. Por otra parte, en la dimensión F, que hace alusión a la interculturalidad, la respuesta de los estudiantes estuvo orientada en un $64,7 \%$ a responder que siempre se da este hecho.

Finalmente, en la dimensión de empleabilidad - $\mathrm{G}$ - el 58,5\% de los estudiantes considera que cursar programas virtuales siempre les ha permitido generar nuevas oportunidades laborales, en consideración a que en la mayoría de los casos los estudiantes se encuentran en regiones consideradas de alta vulnerabilidad y excluidas, indicador que es satisfactorio de acuerdo con la estrategia de hacer presencia en las regiones.

De manera global y casi unánime, se establece que la percepción de los estudiantes gira en torno a reconocer que en la Institución Universitaria Politécnico Grancolombiano siempre y casi siempre, en un 90,5 \%, se promueve la equidad y la inclusión, teniendo en cuenta los criterios propuestos por el Ministerio de Educación Nacional, así como el reconocimiento en la generación de mayores oportunidades laborales gracias a estar inmersos en ambientes virtuales de aprendizaje como parte de su formación en pregrado. 


\section{Discusión}

Este artículo realizó un análisis frente a la percepción que los estudiantes de dos asignaturas de programas virtuales del Politécnico Grancolombiano tienen acerca de la promoción de la equidad y la inclusión desde sus programas académicos de educación superior. La medición exploratoria realizada a 200 estudiantes de regiones vulnerables presenta resultados importantes en torno a la promoción de la equidad y la inclusión en los programas virtuales.

En primera instancia, es necesario resaltar que, frente a las dimensiones seleccionadas para el estudio, existe una actitud hacia el reconocimiento de los esfuerzos institucionales para promover dentro del quehacer educativo las prácticas de equidad e inclusión. Esto coincide con lo propuesto por Raguindin, Ping, Duereh y Lising (2020), quienes afirman que existe una positiva correlación entre factores tales como participación, diversidad y pertinencia, desde una perspectiva de entendimiento de los profesores y las acciones de la administración educativa.

Desde una dimensión pedagógica, se considera que existe comunicación y socialización entre personas de diversas culturas, promoviendo la interacción sobre lo que se considera un cambio cultural para dar paso a la vinculación de personas diversas y el desarrollo de acciones colectivas basadas en un paradigma de cambio, lo que promueve también SilvaLaya (2019) específicamente como un proceso que fortalece a los estudiantes y contribuye la generación de comunidades a través de procesos de transformación cultural.

Por medio de la formación virtual se generan mayores oportunidades para la empleabilidad y el mejoramiento de las condiciones de vida de la comunidad estudiantil, que, de acuerdo con lo expresado por Thielen y Neeser (2020), en su estudio acerca de equidad en el trabajo, es el inicio para la aplicación de los principios de diversidad, 
equidad, inclusión y accesibilidad en el interior del trabajo, con un énfasis en que las organizaciones deben hacer un esfuerzo mayor por promover en su interior el otorgamiento de puestos de trabajo inclusivos y dejando de lado lo tradicional, al permitir vincular personas de acuerdo con sus habilidades, su conocimiento y experiencia, algo que en nuestra cultura aún se está transformando y construyendo.

El Politécnico Grancolombiano cuenta con programas académicos de modalidad virtual que pretenden aportar a la equidad y la inclusión, al brindar la oportunidad de acceso a educación superior a personas de regiones excluidas y vulnerables del país. De ahí que la institución desarrolle estrategias inclusivas como un proceso de admisión flexible y abierto, adaptación de los currículos para que la población excluida pueda desarrollar su pregrado de acuerdo con su ritmo de aprendizaje y un programa de becas que le brinda la oportunidad a los estudiantes de escasos recursos de acceder a programas de educación superior, presencial o virtualmente.

\section{Conclusiones}

Según la Unesco, la educación es un derecho fundamental de todas las personas. En ese sentido, un país como Colombia enfrenta una tarea importante en el mejoramiento de la cobertura y la calidad de la educación. Por otra parte, la inclusión social y la equidad son temas que cada día toman mayor fuerza en un entorno globalizado. Es por esto que la población considerada vulnerable, generalmente, son quienes sufren mayor exclusión social, laboral y educativa; en Colombia, la población vulnerable y excluida se encuentra en zonas rurales y en departamentos como Chocó, Guainía, La Guajira, Cauca, Magdalena y Sucre. En suma, la concentración de las instituciones educativas se da en grandes ciudades como Bogotá, Medellín y Cali, lo que dificulta aún más que las personas de 
regiones apartadas accedan a la educación presencial; en consecuencia, la educación virtual se presenta como una alternativa para la inclusión social.

El Politécnico Grancolombiano cuenta con programas virtuales que han contribuido a la inclusión y educación de personas de regiones como Santander, Amazonas, Arauca, Casanare y Putumayo, entre otras, lo cual evidencia el gran impacto de la institución en la población vulnerable y excluida. La institución cuenta con favorabilidad en la percepción de procesos de intervención, generación de ideas, promoción de la diversidad, oportunidades adecuadas y convenientes, orientación a la excelencia y satisfacción de necesidades, e inclusión de las personas de acuerdo con su mérito con sumo equilibrio e imparcialidad, de modo que se promueve, finalmente, la interacción y la comunicación entre personas de culturas diversas, en proporciones superiores al $90 \%$.

El escenario presentado por el Politécnico Grancolombiano como líder en educación superior virtual equitativa e inclusiva brinda un aporte valioso en la investigación científica para que más instituciones de educación superior que oferten programas virtuales diseñen e implementen actividades y estrategias que promuevan la equidad y la inclusión, especialmente en tiempos como los que está afrontando el país, en los que, debido a la pandemia generada por el Covid-19, ha quedado en evidencia la falta de oportunidades y de acceso a la educación de muchos jóvenes de regiones apartadas de Colombia. De esta manera, habrá más instituciones de educación superior que contribuyan a disminuir las brechas de desigualdad y acceso a la educación, por medio de sus programas académicos virtuales. 


\section{Referencias}

Arias-Vera, J.; Franco-Blanco, L. D.; Rojas-Quitian, M.; Sánchez-Castellanos, M. (2018). El aprendizaje feliz como filosofía institucional: una estrategia de aprendizaje en red en la unidad virtual de la Universidad Manuela Beltrán. Conferencia presentada en el XXI Congreso Internacional EDUTEC 2019. Universitat de Lleida, Lérida, España, 24-26 de octubre. Recuperado de https://bit.ly/3kFtOmk

Arroyo-González, M. J. (2013). La educación intercultural: un camino hacia la inclusión educativa. Revista de Educación Inclusiva, 6(2), 144-159.

Beltrán-Villamizar, Y. I.; Martínez-Fuentes, Y. L.; Vargas-Beltrán, Á. S. (2015). El sistema educativo colombiano en el camino hacia la inclusión. Avances y retos. Educación y Educadores, 18(1), 62-75. DOI: https://doi.org/10.5294/edu.2015.18.1.4

Cabrero-Almenara, J. (2016). La educación a distancia como estrategia de inclusión social y educativa. Revista Mexicana de Bachillerato a Distancia, 8(15), 138-147. DOI: https://doi.org/10.22201/cuaed.20074751e.2016.15.57384

Calvo, G. (2018). Pedagogías inclusivas para los jóvenes: dos modelos flexibles en Colombia. Revista Latinoamericana de Educación Comparada, 9(14), 54-67.

Chona-Duarte, G. (1998). Problemática educativa en Colombia. El papel del profesor, lo que nos compete. Tecné, Episteme y Didaxis: Revista de la Facultad de Ciencia y Tecnología, (4), 41 47. DOI: https://doi.org/10.17227/ted.num4-5690

Departamento Administrativo Nacional de Estadística. (2019). Resultados censo nacional de población y vivienda 2018. Recuperado de https://bit.ly/3u8s/YF

Desigualdad extrema (11 de marzo de 2011). Semana. Recuperado de https://bit.ly/3CIFWJ9

Durán-Gisbert, D.; Giné-Giné, C. G. (2017). La formación del profesorado para la educación inclusiva: un proceso de desarrollo profesional y de mejora de los centros para atender la diversidad. Revista Latinoamericana de Educación Inclusiva. 5(2), 153-170.

Echeita-Sarrionandia, G. (2008). Inclusión y exclusión educativa. Voz y quebranto. Revista Iberoamericana sobre Calidad, Eficacia y Cambio en Educación, 6(2), 9-18.

García, S.; Maldonado, D.; Rodríguez, C. (2014). Propuestas para el mejoramiento de la calidad de la educación preescolar, básica y media en Colombia. Bogotá: Fedesarrollo. Recuperado de https://bit.ly/2XKc2Ft 
Guerrero-Dávila, E. S.; Páramo-Herrera, S. D. (2015). Educación superior inclusiva: una aproximación al diseño de aulas asistivas. Revistas Horizontes Pedagógicos, 17(1), 104-117. Recuperado de https://bit.ly/3kBdCSO

Gutiérrez-Ortiz, A.; García, M.; Barrientos-Monsalve, E. J.; Jejen, J. S. (2018). Factores determinantes en la deserción estudiantil FESC en el programa de Diseño y modas. Convicciones, 5(10), 48-54. Recuperado de https://bit.ly/2XP6jOk

Hernández, J. M.; Villa, L. G.; Vázquez, J. (2017). Ejercicio docente y mediación tecnológica. Memorias del Encuentro Internacional de Educación a Distancia, 5(5), 1-25.

Hurtado-Lozano, L. T.; Agudelo-Martínez, M. A. (2014). Inclusión educativa de las personas con discapacidad en Colombia. CES Movimiento y Salud, 2(1), 45-55. Recuperado de https://bit.ly/3zDKfno

Loaiza-Álvarez, R. (2017). Análisis de los procesos de formación adaptativa en ambientes bimodales para la inclusión social de poblaciones reticentes a través de las TIC. Certiuni Journal, (3), 121-129.

Materón-Palacios, S. (2016). Principios de equidad e igualdad: una perspectiva inclusiva para la atención educativa de las poblaciones con discapacidad en Colombia. Revista Colombiana de Bioética, 11(1), 117-131. DOI: https://doi.org/10.18270/rcb.v11i1.1817

Ministerio de Educación Nacional. (2005). Lineamientos de política para la atención educativa a poblaciones vulnerables. Bogotá: MEN. Recuperado de https://bit.ly/3IWwFXh

Ministerio de educación Nacional. (9 de julio de 2009). Educación virtual o educación en línea. Recuperado de https://bit.ly/3CFThSH

Ministerio de Educación Nacional. (s.f.). La deserción escolar. Recuperado de https://bit.ly/3m0Ca6t

Ministerio de Salud. (s. f.). Poblaciones vulnerables. Recuperado de https://bit.ly/2XYUiqf

Mondragón-Hernández, A. (2018). Educación inclusiva y virtualidad: aplicación del Índice de Inclusión para la Educación Superior a un programa virtual. Revista Docencia Universitaria, 19(1), 75-96. Recuperado de https://bit.ly/3IVCt3f

Murillo, J.; Duk, C. (2018). Una Investigación inclusiva para una educación inclusiva. Revista Latinoamericana de Educación Inclusiva, 12(2), 11-13. DOI: https://doi.org/10.4067/S0718$\underline{73782018000200011}$

Navarro, M.; Martínez de Anguita, P. (2008). Blend learning para la educación rural: ¿una alternativa para Colombia? Escuela Abierta, 11(1), 29-48. Recuperado de https://bit.ly/3u7OITD 
Perfetti, M. (2004). Estudio sobre la educación para la población rural en Colombia. En FAO (Ed.) Educación para la población rural en Brasil, Chile, Colombia, Honduras, México, Paraguay y Perú. (164-216). Proyecto FAO-UNESCO-DGCS/ITALIA-CIDE-REDUC. Recuperado de https://bit.ly/3hZZIrR

Politécnico Grancolombiano. (24 de septiembre de 2020). Proyecto Educativo Institucional. Recuperado de https://bit.ly/3CO8YHz

Raguindin, P. Z.; Ping, L. Y.; Duereh, F.; Lising, R. L. (2020). Inclusive practices of in-service teachers: a quantitative exploration of a southeast Asian context. European Journal of Educational Research, 9(2), 787-797. DOI: https://doi.org/10.12973/eu-jer.9.2.787

Rojas-Rojas, L. M.; Arboleda-Toro, N.; Pinzón-Jaime, L. J. (2018). Caracterización de población con discapacidad visual, auditiva, de habla y motora para su vinculación a programas de pregrado a distancia de una universidad de Colombia. Revista Electrónica Educare, 22(1), 1 28. DOI: https://doi.org/10.15359/ree.22-1.6

Rueda-Ortiz, R.; Franco-Avellaneda, M. (2018). Políticas educativas de TIC en Colombia: entre la inclusión digital y formas de resistencia-transformación social. Pedagogía y Saberes, (48), 9-25. DOI: https://doi.org/10.17227/pys.num48-7370

Silva-Laya, M. (2019). Tres iniciativas de equidad educativa universitaria en la Ciudad de México. Revista Mexicana de Investigación Educativa, 24(80), 43-68. Recuperado de https://bit.ly/2XOSU9L

Thielen, J.; Neeser, A. (2020). Making job postings more equitable: evidence based recommendations from an analysis of data professionals job postings between 2013-2018. Evidence Based. Library and Information Practice, 15(3), 103-156. DOI: https://doi.org/10.18438/eblip29674

Unesco. (2019). Informe de seguimiento de la educación en el mundo 2019. Informe sobre género: Construyendo puentes para la igualdad de género. París: Unesco. Recuperado de https://bit.ly/3CMcLoS

Vilanova, G. (2016). Modelo pedagógico emergente en la formación mediada por tecnología de ingenieros en el ámbito universitario. En N. Callaos; B. Sánchez; A. Tremante; G. Vilanova; F. Welsch (Eds.) Memorias de la Décima Quinta Conferencia Iberoamericana en Sistemas, Cibernética e Informática. (239-244). Florida: International Institute of Informatics and Systemics. Recuperado de https://bit.ly/2ZtBIMO 